\title{
MAGNETO-OPTICAL RECORDING WITH OPTICAL AND MAGNETIC FIELD MODULATION METHODS
}

\author{
Y. MURAKAMI, A. TAKAHASHI, K. OHTA and T. ISHIKAWA \\ Precision Tech. Lab., Sharp Corp., 2613-1 Ichinomoto-cho, Tenri, Nara 632, Japan
}

\begin{abstract}
The magneto-optical recording characteristics of three types of DyFeco films were measured using three recording methods; the optical modulation method, the magnetic field modulation method and the laser off method in which the laser power was lowered only while the magnetic field was changing. The laser off method improved the $\mathrm{C} / \mathrm{N}$ in the low external magnetic field. Moreover, the erasability in the laser off method was investigated, and the value of -30dB was achieved.

KEYWORDS: MAGNETO-OPTICAL DISK, MAGNETIC FIELD MODULATION METHOD, OPTICAL MODULATION METHOD, TRANSITION METAL RICH, RARE EARTH METAL RICH, COMPENSATION, CARRIER TO NOISE RATIO, ERASABILITY
\end{abstract}

\section{INTRODUCTION}

Magnetic field modulation methods have been studied as a direct overwrite for magneto-optical disks and two typical recording methods have been proposed. One of them uses a continuous laser irradiation and the other uses a pulsed laser irradiation synchronized with a channel clock [1], [2].

In this paper, we report a new overwrite method (laser off method) in which the laser is lowered only while a magnetic field is switched. Using the laser off method and two recording methods ; a conventional magnetic field modulation method (overwrite mode using a continuous laser irradiation) and an optical modulation method (not overwrite mode), we examined recording and erasing characteristics of DyFeCo films of three different compositions. The difference of the external magnetic field dependence of the carrier-to-noise ratio $(\mathrm{C} / \mathrm{N})$ in above-mentioned three recording methods, and the erasing characteristics in the laser off method were reported.

EXPER IMENT

Fig. 1 shows the medium structure and the recording test configuration.
In the quadrilayer structure, a DyEeCo magnetic layer with $20 \mathrm{~nm}$ thickness was sandwitched between AIN layers and covered with an Al reflective layer. These films were prepared by sputtering on a polycarbonate disk with a 3.5 inch diameter and reflectivity of the medium was 17\%. In this structure, three different films Dy $\left(\mathrm{Fe}_{78} \mathrm{CO}_{22}\right)_{1-x}$, $x=0.21,0.23,0.24$ were prepared. The compositions were determined by $x$-ray fluorescence analysis.

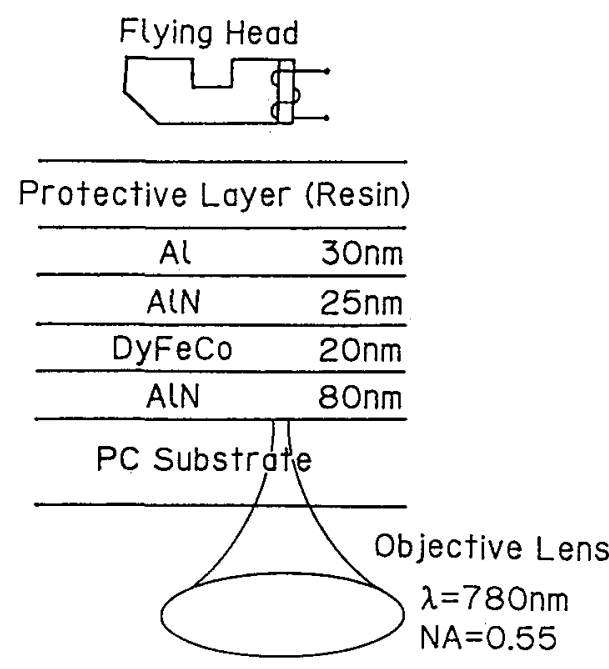

Fig. 1 Medium structure and recording test configuration. 
Fig. 2 shows coercive force and Kerr rotation angle as a function of temperature for disks $(A, B, C)$. In the disk $B$, the film has the compensation temperature of 20 to $30 \%$ and the Curie temperature of $210 \mathrm{C}$. In comparison with the disk B, the disk A is transition metal rich (TM rich) and the disk $C$ is rare-earth metal rich (RE rich) respectively. To prevent magnetic head crash and also protect against oxidation and corrosion of recording medium, a UV cured resin layer with about $10 \mu \mathrm{m}$ thickness was coated on the AI reflective layer. The spacing between the head and the UV resin surface was about $3 \mu \mathrm{m}$ when the linear velocity was $10 \mathrm{~m} / \mathrm{sec}$. Fig. 1 also shows the optics configuration for this experiment. Wavelength of the laser diode was $780 \mathrm{~nm}$ and numerical aperture of the focusing lens was 0.55 . Under these conditions, we measured recording characteristics of the disks using next three recording methods.

(1) a conventional optical modulation method. (not overwrite mode)

(2) a conventional magnetic field modulation method. (overwrite modé using a continuous laser irradiation)

(3) the laser off method. (overwrite mode)

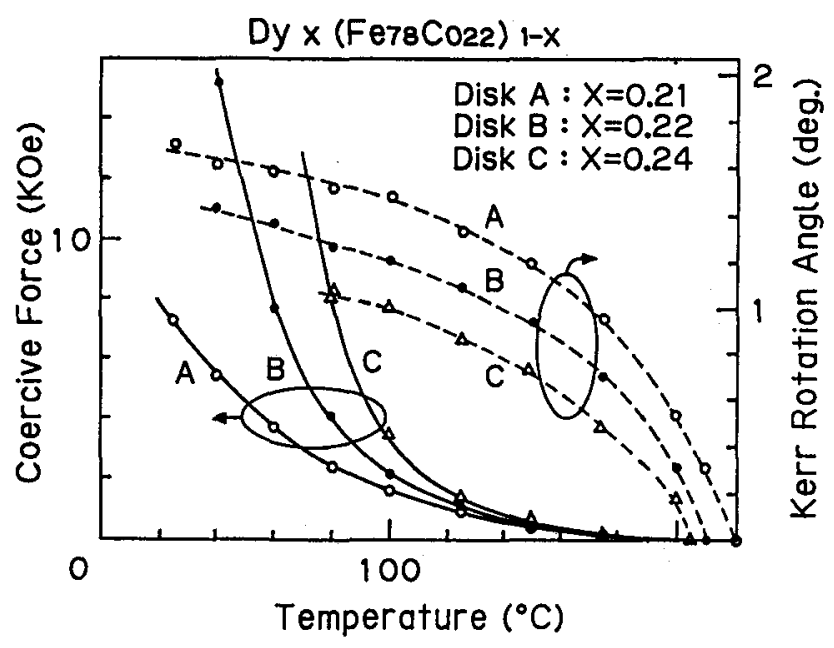

Fig.2 Temperature dependence of coercive force and Kerr rotation angle of DyEeCo films.
Fig. 3 shows the timing chart of laser power and magnetic field in the laser off method. The laser power was lowered while the magnetic field was switching. In this experiment, the value of the low laser power was set at $1 \mathrm{~mW}$. The value corresponded to that of the read power. The switching time of magnetic field was $25 \mathrm{nsec}$ from -2000 e to +2000 .



Fig.3 Timing chart of laser beam and magnetic field in the laser off method.

\section{RESULTS AND DISCUSSION}

Dependence of $\mathrm{C} / \mathrm{N}$ on external magnetic field

Fig. 4 shows the dependence of $\mathrm{C} / \mathrm{N}$ on the external magnetic field (Hex). In Fig.4, A, B and C indicate the disk A, B and $C$ shown in Fig. 2 respectively. The recorded bit length was about $0.9 \mu \mathrm{m}$, and the recording frequency was $5.6 \mathrm{MHz}$. In every disk and every recording method, the value of the optimum write laser power which gave rise to the best $\mathrm{C} / \mathrm{N}$ at Hex $=1200$ e was $8 \mathrm{~mW}$. In the conventional optical modulation method(Fig.4 (a)), the $\mathrm{C} / \mathrm{N}$ of the disk $\mathrm{A}$ was higher and the Hex for the saturated $\mathrm{C} / \mathrm{N}$ of the disk $A$ was lower than that of the other disks. The $\mathrm{C} / \mathrm{N}$ value of the disk $\mathrm{C}$ was lowest because of the low curie temperature. Using the conventional magnetic field modulation method(Fig.4 (b)), the writing noise increased for all disks in comparison with the optical modulation method and the $\mathrm{C} / \mathrm{N}$ of all disks decreased. However, using the laser off method (Fig. 4 (c)), the $\mathrm{C} / \mathrm{N}$ of all disks increased and were nearly equal to that in the optical modulation method. The $\mathrm{C} / \mathrm{N}$ of the disk $\mathrm{A}$ was saturated when Hex was over 800 . 

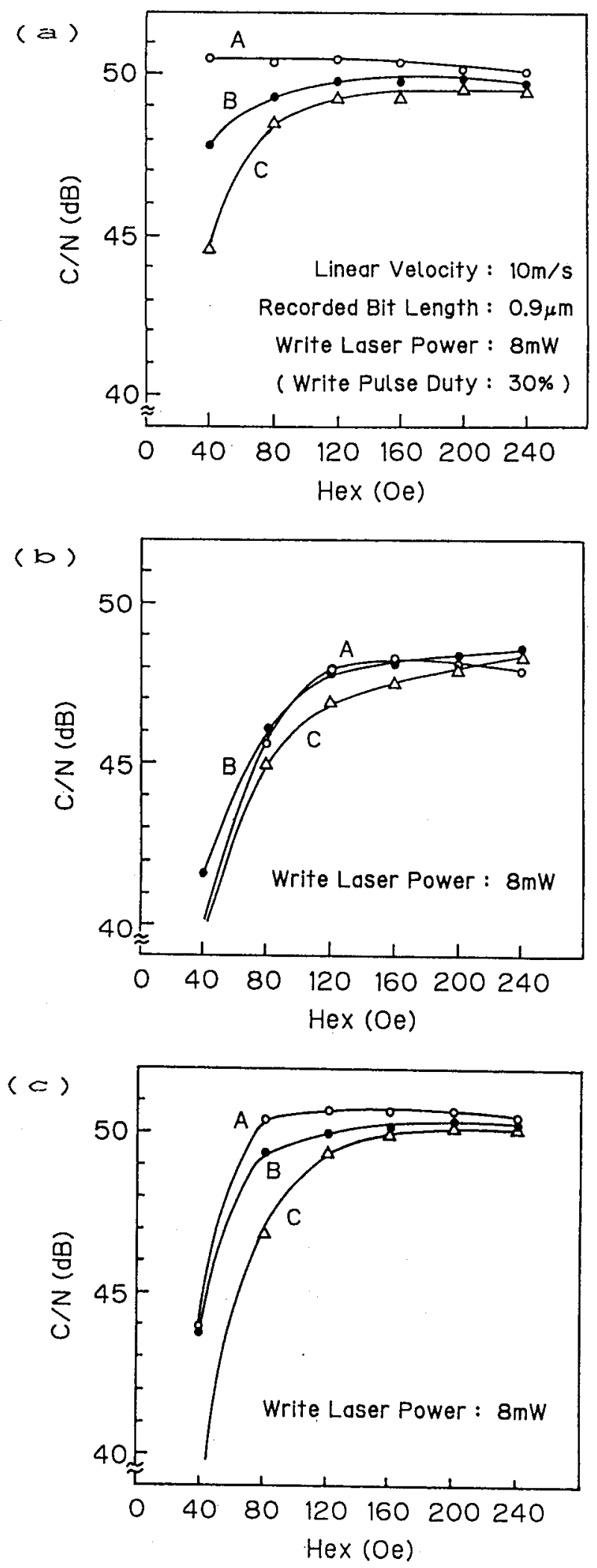

Fig. 4 Dependence of carrier-to-noise ratio $(\mathrm{C} / \mathrm{N})$ on external magnetic field(Hex) using three recording methods: (a) the conventional optical modulation, (b) the conventional magnetic field modulation, (c) the laser off method.
The disk A had a TM rich composition film so that magnetic field from the surrounding media when bits are recorded is larger than that in the other disks. This is one of the reasons why the Hex for saturated $C / N$ of the disk $A$ was lower than that in the other disks in the conventional optical modulation method. On the other hand, in the conventional magnetic field modulation method, the above-mentioned magnetic field from the surrounding media causes the irregularity of the recording bits when the external magnetic field was changing. Thus the write noise increased and the $\mathrm{C} / \mathrm{N}$ decreased for all disks, especially in low Hex region. In the laser off method, the laser power was modulated between high and low levels so that the temperature profile of recording medium was close to that in the conventional optical modulation method. Therefore, the $\mathrm{C} / \mathrm{N}$ value and the dependence on Hex in the laser off method was likely to those in the conventional optical modulation method. In order to substantiate the results, the magnetic domains recorded using three recording methods were observed with a polarized microscope. The shape of domain recorded in the conventional magnetic field modulation method was like a

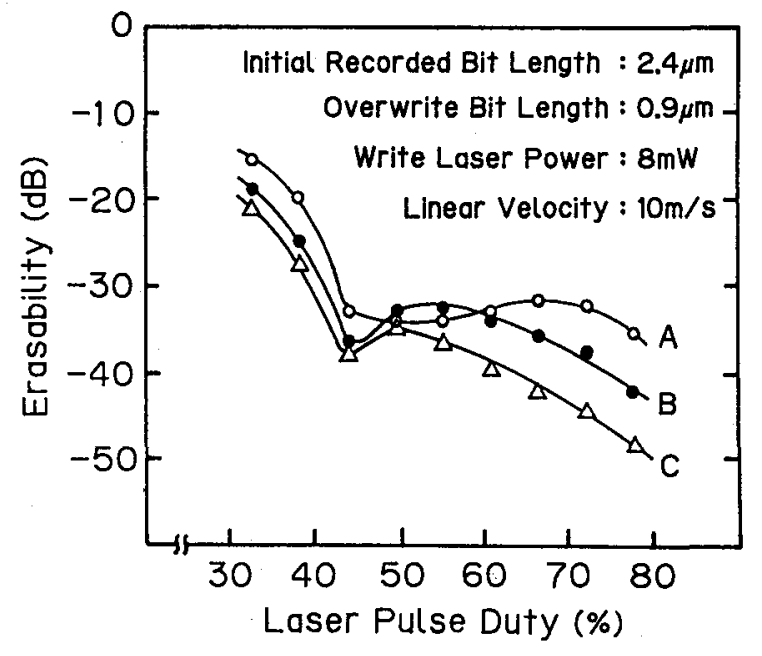

Fig.5 Dependence of erasability on write laser pulse duty using the laser off method. (when a $2.1 \mathrm{MHz}$ signal was overwritten by a $5.6 \mathrm{MHz}$ signal) 
chevron. In the laser off method, the shape was like a circle which, was similar to that in the optical modulation method.

Erasability in the laser off method

Next, erasability of three disks in the laser off method was measured. In the method, it is important to know the overwrite characteristics because there is some area where the laser is not irradiated. In this experiment, the difference of the carrier level of the pre-recorded 2.1 MHz signal between before and after overwriting of $5.6 \mathrm{MHz}$ signal was defined as the erasability. Fig. 5 shows the dependence of the erasability on the write laser pulse duty in the laser off overwrite method. The write laser power, Hex and the linear velocity were $8 \mathrm{~mW}, 1600$ and $10 \mathrm{~m} / \mathrm{s}$ respectively. $\mathrm{C} / \mathrm{N}$ values of the initial $2.1 \mathrm{MHz}$ signal were about $56 \mathrm{~dB}$ in every disk. For all disks, the erasability over -30dB was achieved when the write laser pulse duty was over $45 \%$ and the value is considered to be good enough for practical use.

\section{CONCLUSION}

As a direct overwrite method for magneto-optical disks, the laser off overwrite method was examined. Using the method, the read-out signal quality was improved in comparison with the conventional magnetic field modulation using a continuous laser irradiation. Moreover, the recording media with a transition metal rich composition of DyFeCo exhibited good $\mathrm{C} / \mathrm{N}$ in the low magnetic field.

\section{REFERENCES}

[1] T.Nakao, et al., International Symposium on Optical Memory Technical Digest p.5 (1987).

[2] T.Watanabe and H.Ogawa, Digests of Optical Memory Symoposium '88, p.47 (1988) . (in Japanese) 